\title{
A Problem for the 21st/22nd Century
}

Sylvain Crovisier (CNRS and Université Paris-Sud, France) and Samuel Senti (Universidade Federal do Rio de Janeiro, Brazil)

Sylvain: At the end of 1997, Jean-Christophe welcomed me in his huge office at the Collège de France. I had already met him two years earlier at an oral "concours". This time I came to ask him to supervise my DEA thesis. On the board he talked at length about dynamical systems, which he presented with a diagram.

\author{
QUASI-PERIODIC \\ SYSTEMS \\ small-divisors $\mathcal{E}$ KAM theory
}

He had already done a lot of work on quasi-periodical systems (circle diffeomorphisms, rational maps), but in recent years he was more interested in (non-uniformly) hyperbolic systems; a very hot topic. I left full of enthusiasm, with his lecture notes on Jakobson's Theorem.

Samuel: My experience is quite similar to yours. Two years earlier, the inaugural course at the Collège de France on Jakobson's theorem had not been held yet. Still the same discussion took place, with the same supporting diagram, a wellknown schema to all those who had the chance to see JeanChristophe expose his programme: a short, medium and longterm programme, and even a very long-term programme since he dared not hope to fulfill it during his career.

We had the opportunity to follow a little bit of JeanChristophe's path in the non-uniformly hyperbolic world, and to hear him share his dream of one day understanding conservative systems. In these few pages we try to give an overview of that dream.

\section{Hyperbolic dynamics}

The study of the dynamics of a map $f$ from a space $X$ to itself consists in the description of its orbits, i.e., of sequences of the form $x, f(x), f(f(x)), f(f(f(x))), \ldots$ We denote by $f^{n}(x)$ the $n^{\text {th }}$ iterate of $x$.

\section{Uniform hyperbolicity in dimension one}

Let us start by describing the dynamics of the quadratic map $f_{a}: x \mapsto x^{2}+a$ on the real line for parameter values $a<-2$. We observe two kinds of orbits: some go to infinity; the others belong to an invariant Cantor set K. Moreover, for all points $x \in K$ the sequence of iterated derivatives $D f_{a}^{n}:=D f_{a} \circ \cdots \circ$ $D f_{a}(x)$ grows exponentially at a given uniform rate which is independent of $x$.

Despite the system's apparent simplicity, describing the dynamics turns out to be a surprisingly complex task for parameter values $-2<a<0$. Indeed, denoting by $\beta_{a}$ the positive fixed point of $f_{a}$, the interval $\left[-\beta_{a}, \beta_{a}\right]$ turns out to be invariant and the orbit of the critical point 0 remains bounded. For some values of the parameter (for instance for $a=-0.8$ ), one can decompose the system as follows:

- The orbit of any point outside $\left[-\beta_{a}, \beta_{a}\right]$ goes to infinity.

- There is an invariant compact set $K$ which does not contain 0 .



Figure 1. Orbit of a uniformly hyperbolic map

- There is an attractive periodic orbit which attracts the orbit of any point in $]-\beta_{a}, \beta_{a}[\backslash K$.

This dynamic is uniformly hyperbolic: for orbits contained in $K$, the sequence of iterated derivatives $\left|D f_{a}^{n}\right|:=\mid D f_{a} \circ \ldots$ ○ $D f_{a}$ l grows exponentially at a uniform rate; on the other hand, for any other bounded orbit, it decreases to 0 exponentially.

Hyperbolicity is a fundamental property: it implies that the orbits of any two points in $K$, however close, always end up being distinct from one another. We can thus associate to each orbit a sequence in $\{-1,1\}$ which encodes the sign of each iterate. Furthermore, hyperbolicity implies the system's stability: for any mapping $C^{1}$-close to $f_{a}$, the structure of the space of orbits is unchanged.

\section{Non uniform hyperbolicity}

In 1981 Jakobson [6] showed that there exists another typical dynamical behavior:

Theorem (Jakobson). There is a set of parameters of nonzero measure $\mathcal{P} \subset[-2,0]$, such that for $a \in \mathcal{P}$ and almost all $x \in\left[-\beta_{a}, \beta_{a}\right]$,

- the orbit of $x$ for the quadratic map $f_{a}$ is dense in the interval $[a, a(a+1)]$,

- the asymptotic growth rate of the sequence of iterated derivatives $\left|D f_{a}^{n}(x)\right|$ is exponential.

The dynamics of the maps $f_{a}$ is then rather well understood: the orbit of almost every point of the interval $\left[-\beta_{c}, \beta_{c}\right]$ equidistributes to the same measure (which has support in $[a, a(a+1)])$; outside that interval all orbits escape to infinity. The dynamics is non-uniformly hyperbolic: we observe an increase of the derivatives along the orbit of almost all points $x$, but that exponential growth is no longer uniform in $x$, since some orbits can land more or less close to the critical point 0 where the derivative is zero.

The proof of Jakobson's theorem is delicate: although the behaviour described has non-zero measure in $[-2,0]$, its complement is dense: this is Fatou's conjecture, proved by Graczyk and Świątek, as well as by Lyubich in the 1990s. 


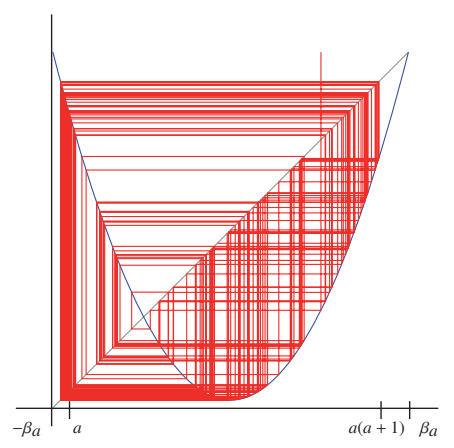

Figure 2. Orbit of a non-uniformly hyperbolic map

Afterwards, Rees [11] extended this result to rational maps on the Riemann sphere.

\section{Uniform hyperbolicity on surfaces}

For surface diffeomorphisms, the horseshoe and Plykin's attractor are typical examples of hyperbolic dynamics. Now the following property $(\mathrm{H})$ holds at every point $z$ :

- $\left(D f^{n}(z)\right)_{n \geq 0}$ exponentially expands vectors outside a subspace $E^{s}(z)$.

$(\mathcal{H}) \circ\left(D f^{-n}(z)\right)_{n \geq 0}$ exponentially expands vectors outside a subspace $E^{u}(z)$.

- $E^{s}(z), E^{u}(z)$ span the tangent space at $z$.

And so, there is a uniform integer $N \geq 1$ such that for every $z$ and every non zero vector $v$, the image $D f^{N}(z) \cdot v$ or $D f^{-N}(z) \cdot v$ has norm bigger than $2\|v\|$. Again, we can decompose the dynamics as follows: there is a finite collection of elementary invariant compact sets onto which past and future orbits accumulate. There are three different types of such sets:

- Subsets that attract all orbits originating from a neighbourhood are the attractors. They can be periodic attractive orbits (sinks), or laminated sets, expanded by the dynamics, of which Plykin's attractor is an example.

- Subsets attracting every past orbit originating from a neighbourhood are the repellers.

- There are also saddle-like subsets, in the neighbourhood of which some orbits escape by future and past iterations. The horseshoe is an example of such a set.

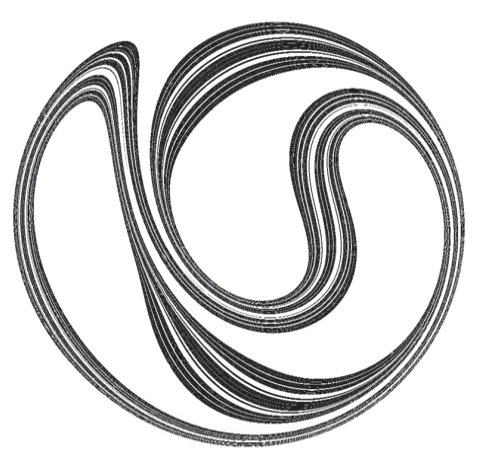

Figure 3. A Plykin attractor. It carries a lamination by curves. The dynamics expands along the leaves and contracts in the transversal direction.

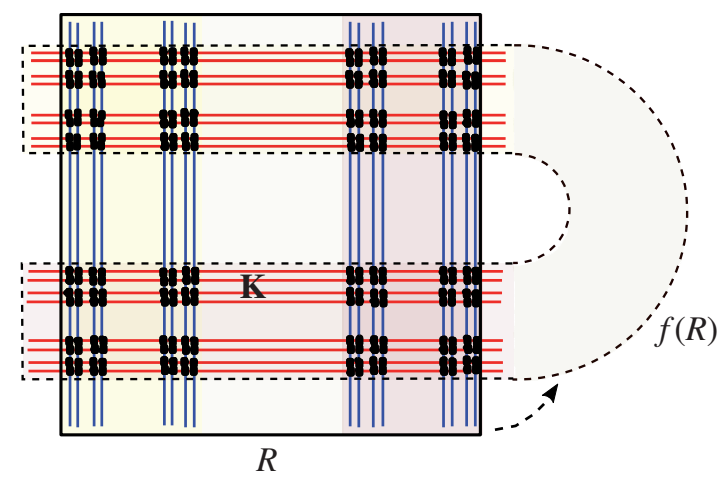

Figure 4. The horseshoe: A Cantor set $K$ having a product structure. The dynamics stretches and folds a rectangle $R$, contracting in the vertical directions and expanding in the horizontal ones.

To learn more about uniformly hyperbolic dynamics we strongly recommend the introductory text written by JeanChristophe [16].

\section{Non uniform hyperbolicity on surfaces}

Hyperbolic diffeomorphisms were extensively studied in the 1960-70s, but it soon became clear that they were not sufficient to describe most dynamics. In a 1991 paper, Benedicks and Carleson [1] generalised Jakobson's theorem to surface diffeomorphisms. They studied the Hénon family, i.e. diffeomorphisms of the plane of the form:

$$
f_{a, b}:(x, y) \mapsto\left(x^{2}+a-y, b x\right) .
$$

Theorem (Benedicks-Carleson). There is an open region $U$ of the plane and a set of parameters $\mathcal{P} \subset \mathbb{R}^{2}$ of non-zero measure, such that for every $(a, b) \in \mathcal{P}$ there is a compact set $\Lambda_{a, b} \subset U$ satisfying:

- $\Lambda_{a, b}$ is an attractor of the Hénon map $f_{a, b}$ : the future orbit of every point in $U$ accumulates on a part of $\Lambda_{a, b}$,

- $\Lambda_{a, b}$ is transitive: it contains a point $z$ whose orbit is dense in $\Lambda_{a, b}$,

$-\Lambda_{a, b}$ is not a periodic orbit.

Subsequent work by Benedicks-Young [3] and BenedicksViana [2] showed that orbits of almost all points of $U$ distribute towards the same probability measure $\mu_{a, b}$, supported on the attractor $\Lambda_{a, b}$. Property $(\mathcal{H})$ stated previously is satisfied, asymptotically, $\mu_{a, b}$-almost everywhere. It is not satisfied everywhere because $U$ 's topology is an obstruction to uniform hyperbolicity.

Benedicks and Carleson's attractor is a "non-uniform version" of Plykin's attractor. The existence proof of such systems is a tour de force: indeed, Newhouse [8] showed that arbitrarily close to the parameters identified by Benedicks and Carleson there are parameters for which future orbits from $U$ split into a infinite family of different attractors. Non uniform hyperbolicity translates into the existence of critical points, i.e. points $z \in \Lambda_{a, b}$ admitting a vector $v \neq 0$ such that $\left\|D f_{a, b}^{n}(z) \cdot v\right\|$ decreases exponentially when $n$ goes to $+\infty$ as well as to $-\infty$. Homoclinic tangencies are simple examples of critical points: in that case, $z$ is a tangency point between two curves $W^{s}$ (called stable) and $W^{u}$ (called unstable) having the property of being contracted towards the same periodic orbit $O$ both by positive respectively negative iterations. The 


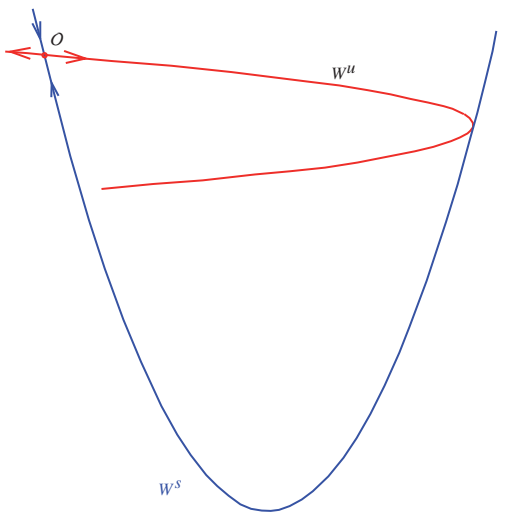

Figure 5. An homoclinic tangency between the stable, $W^{s}$, and unstable, $W^{u}$, curves of a fixed point $O$

presence of critical points makes the analysis of the dynamics especially difficult.

\section{Non-uniformly hyperbolic horseshoes}

Jean-Christophe quickly followed the development of non uniformly hyperbolic dynamics: in 1990 he gave a Bourbaki seminar [18] where he presented the work of Jakobson, Rees and Benedicks-Carleson. His 1994 talk [17] at the International Congress of Mathematicians in Zürich underlines the importance of this new topic. It is also the subject of a course which he gave at the ETHZ in 1996, of his inaugural lesson in 1997, and then of six of his courses at the Collège de France between 1998 and 2016.

His goal is clearly stated [17]:

What we would like to do in the next few years is to obtain a conceptual theory of "weakly hyperbolic basic sets" (including of course the striking examples considered above). For a smooth diffeomorphism $f$ of a manifold $M$, such a "weakly hyperbolic basic set" should again be a compact, invariant, transitive, locally maximal subset $K$ of $M$ satisfying moreover some kind (?) of weak hyperbolicity condition.

Several aspects of the non-uniformly hyperbolic sets described by Benedicks and Carleson in [1] and of their methods deserve to be mentioned. First of all, future iterations are favoured over past iterations: in this framework only the first condition in $\mathcal{H}$ is hard to obtain. Finally, in the proof we need to assume that the dynamics is extremely dissipative (the parameter $b$ - the jacobian - is assumed to be very close to 0 ) so that the attractor is very thin, almost a curve.

Jean-Christophe considers a different approach to the study of non-uniform hyperbolicity, in the spirit of Jakobson's initial proof. As early as 1997-98 his course announces a "general theory" of non-uniformly hyperbolic systems for surface diffeomorphisms. His goal is to develop a technique that is symmetric with respect to time, which is not restricted to attractors, and which also helps us understand sets of large Hausdorff dimension.

Together with Jacob Palis, they propose to apply this programme to the study of homoclinic bifurcations of horseshoes: points whose future or past orbits are attracted by such a hyperbolic set define two laminations (a stable and an unstable one). A change in the dynamics allows the deformation

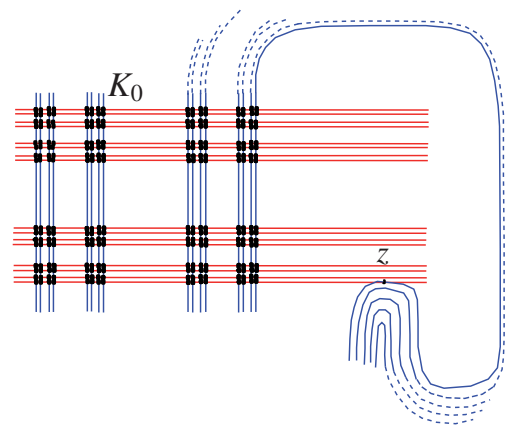

Figure 6. Tangency between the stable and unstable laminations of a horseshoe

of these laminations in order to create new intersections and thus enrich the initial dynamics. When the horseshoe is sufficiently thick some intersections between the leaves of the two laminations produce tangencies that persist for neighbouring systems: this was highlighted by Newhouse [8] at the end of the 1970's, and then made precise by Palis, Takens, Moreira and Yoccoz $[7,9]$.

\section{Palis-Yoccoz theorem}

In this theorem the authors consider a surface diffeomorphism $f_{0}$ with a horseshoe $K_{0}$ and also a quadratic tangency point $z$ between a stable leave and an unstable leave. The tangency is assumed to be isolated: the union $\Lambda_{0}$ of $K_{0}$ with the orbit $z$ has a neighbourhood $U$ from which every orbit originating from $U \backslash \Lambda_{0}$ escapes. For a generic family $\left(f_{t}\right)$ containing $f_{0}$ the goal is to describe the set of points $\Lambda_{t}$ whose orbit remains in $U$ in the future as well as in the past. One can also assume that the stable and unstable laminations still intersect in the neighbourhood of $z$ for parameters $t>0$.

We associate to $K_{0}$ the dimensions $d^{s}$ and $d^{u}$ transversal to the stable and unstable laminations. The Hausdorff dimension of $K_{0}$, equal to $d^{s}+d^{u}$, plays an essential role:

- When $d^{s}+d^{u}<1$, the set of parameters $t>0$ for which the dynamics is hyperbolic [9] has total density at 0 .

- When $d^{s}+d^{u}>1$, the set of parameters $t>0$ for which the dynamics is not hyperbolic [7] has total density at 0 .

Trying to understand how this "loss of hyperbolicity" comes about, Jean-Christophe and J. Palis focused on the case where $d^{s}+d^{u}$ is slightly bigger than 1 . They showed that nonuniform hyperbolicity prevails in an explicit neighbourhood $D_{P Y} \subset\left\{\left(d^{s}, d^{u}\right), d^{s}+d^{u}>1\right\}$ of the locus $d^{s}+d^{u}=1$.

Theorem (Palis-Yoccoz [10]). Fix $\left(d^{s}, d^{u}\right) \in D_{P Y}$. Then for a non-zero measure parameter set $t>0$ the set $\Lambda_{t}$ is a nonuniformly hyperbolic horseshoe.

The notion of "non-uniformly hyperbolic horseshoe" is technical, however it satisfies two essential properties:

- The set of orbits attracted by $\Lambda_{t}$ (in the past or in the future) has zero Lebesgue measure. In particular $\Lambda_{t}$ is "saddle"like and does not contain either an attractor or a repeller.

- Most (in a sense that will not be made precise here!) orbits of $\Lambda_{t}$ equidistribute by future iterations to an invariant probability measure $\mu_{t}^{+}$. Furthermore, $\mu_{t}^{+}$-almost all points satisfy $\mathcal{H}$. (A similar property is satisfied for past iterations, with respect to another measure $\mu_{t}^{-}$.) 


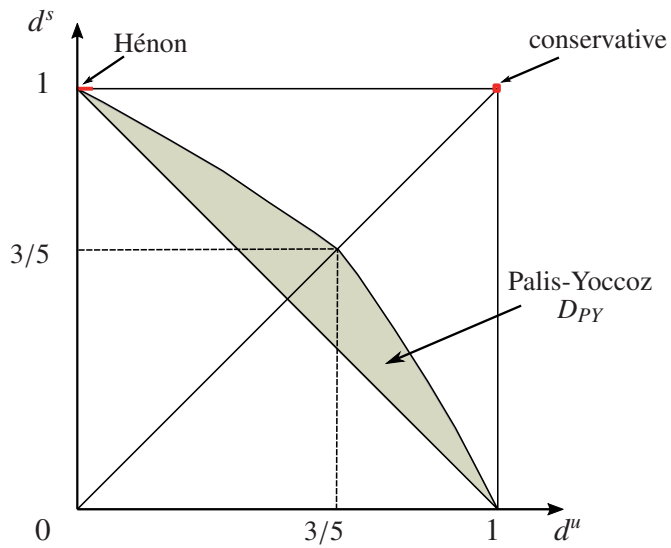

Figure 7. Stable and unstable dimensions of a horseshoe

The proof [10] took several years and fills a whole volume of the Publications Mathématiques de l'IHES. The impact left by Jean-Christophe on this topic is considerable and exceeds by far that monumental article.

\section{Strong regularity}

The analysis of non-uniformly hyperbolic dynamics proposed by Benedicks and Carleson depends on a careful control of the growth of the iterated derivatives at the critical points. However, Jean-Christophe understood that by introducing an $a d$ hoc version of Yoccoz puzzles (see [5], by Xavier Buff) he would be able to isolate a structure allowing one to express the choice of parameters in combinatorial terms. It turns out this structure is sufficiently rigid to lead to the proof of the positivity of the measure of the set of parameters in question. This approach follows the spirit of Jakobson's proof in that the analysis of the dynamics is concentrated on a sequence of uniformly hyperbolic sets whose uniformity degenerates as they approach the critical value.

\section{Strong regularity in one dimension}

In order to understand these ideas in more detail, it is useful to revisit the one dimensional case.

When the dynamics is uniformly hyperbolic, the hyperbolic set $K$ can be covered by a finite family $I_{i}$ of disjoint intervals, each one being diffeomorphically mapped to a predetermined interval by an iterate $f_{a}^{n_{i}}$.

To study the dynamics of the map $f_{a}(x)=x^{2}+a$ when $a \in[-2,0]$, Jean-Christophe [13] considers the interval $A=$ $\left[\alpha_{a},-\alpha_{a}\right]$ where $\alpha_{a}$ is the negative fixed point of $f_{a}$. Again we can try to fill in the interval $A$ with a (this time countable) family $I_{i}$ of intervals. For the simplest intervals (away from the critical point 0 ), all the iterates $f^{k}\left(I_{i}\right)$ are disjoint from $A$ when $1 \leq k<n_{i}$. Near the critical point 0 , there are smaller intervals that can return several times to the interval $A$ before covering it.

This construction has an advantage: it allows for a successive approximation of the dynamics by the set $K$ of orbits that only visit a finite family of intervals $I_{i}$; since $K$ avoids a neighbourhood of the critical point, a result by Mañé shows that it is uniformly hyperbolic. Enriching the family $I_{i}$ degenerates the hyperbolicity.



Figure 8. Yoccoz's course notes on Jakobson's theorem

The decomposition of $A$ in intervals $I_{i}$ allows one to study the dynamics in a combinatorial way, and to code the orbits through the sequence of intervals it visits. To capture the dynamics which are not uniformly hyperbolic one must allow the critical orbit to return ever closer to the critical point. JeanChristophe defined strongly regular maps: these are maps $f_{a}$, for which most returns of the critical orbit to the interval $A$ belong to a simple interval $I_{i}$. The hypothesis that the sum of all "deep" return times of the critical orbit only represent a small fraction of all the iterates, guarantees that the family $I_{i}$ indeed fills almost all the interval $A$, implying that the dynamics is non-uniformly hyperbolic.

\section{Parameter selection}

It remains to show that in the parameter set the strongly regular maps have positive Lebesgue measure. Following Adrien Douady's adage sow in the dynamics space to harvest in the parameters space, Jean-Christophe shows that the dependency of the puzzle structure induces a similar structure in the parameter set, and that the latter structure is slowly varying when one varies the parameter; a transversality phenomenon that allows one to take advantage, in the parameter space, of the estimates obtained in the dynamics space.

For the parameter $a=-2$ the second iteration of the critical point is fixed and all the intervals are simple. As a parameter $a>-2$ gets closer to -2 , the part of $A$ that is not filled by simple intervals is contained in an ever smaller neighbourhood of the critical point. The dynamics in the complement set is uniformly hyperbolic and the estimates on the expansion rate obtained for this set are good. The strong regularity con- 


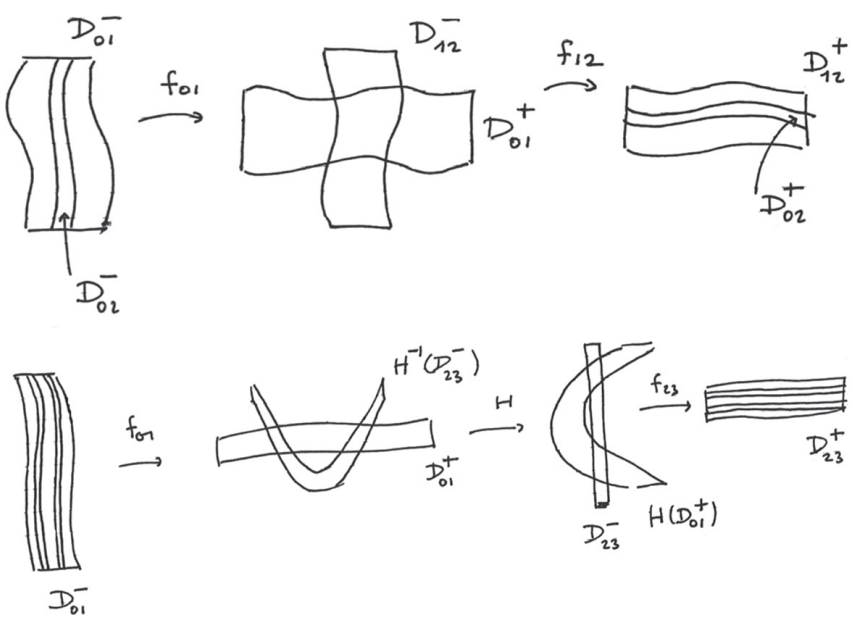

Figure 9. Maps of affine and fold types (Sketch by J.-C. Yoccoz)

dition allows for enough deep returns to exploit those good estimates in the parameter space and prove, by a large deviations argument, that the set of strongly regular parameters has a Lebesgue density point at $a=-2$.

\section{Strong regularity on surfaces}

In higher dimensions, iterating a uniformly hyperbolic surface diffeomorphism leads to composing "affine- like" maps that expand and contract along two transverse directions (see figure 9): the composition of such maps is still affine. However, when critical points are present, one must also consider "foldlike" maps, i.e., maps of quadratic type. We must then check that the compositions that show up in the system preserve this new class of maps. In general this is not the case, and one must select the parameters for which the folding effects do not accumulate by iteration. The shift to dimension two also brings other difficulties. Indeed, the sole critical point $c=0$ is replaced by an infinite critical set: the parameter selection must then take each of its points into account. Furthermore, this set is not known a priori like in dimension 1: the definition of critical point introduced previously requires the analysis of the behaviour of arbitrarily long sequences $\left\|D f^{n}(z) \cdot v\right\|$, and hence a precise enough knowledge of the dynamics.

Since the critical set contains the tangency points between the stable and unstable laminations (as in figure 6), we expect its dimension to be bounded below by $d^{s}+d^{u}-1$, which is the typical dimension for the intersection of Cantor sets. Therefore the critical set is all the more difficult to control, as the dimension of the horseshoe is large. The case of the dynamics of the Hénon attractor studied by Benedicks and Carleson would correspond to a dimension $d^{s}=1$ and a dimension $d^{u}$ extremely close to 0 . By comparison, the hypothesis made on $\left(d^{s}, d^{u}\right)$ by Palis-Yoccoz is completely explicit and allows us to get close to $d^{s}+d^{u}=6 / 5$.

The proof of [10] did not fully meet Jean-Christophe's requirements: the definition of strong regularity that is stated there depends not only on the map, but also on the parametrisation of the family. The concept was taken up and reworked a few years later by Pierre Berger [4] in the context of attractors. This led Jean-Christophe to schedule his 2016 course at the Collège de France on a proof of Benedicks and Carleson's theorem which uses strong regularity and which relies heavily

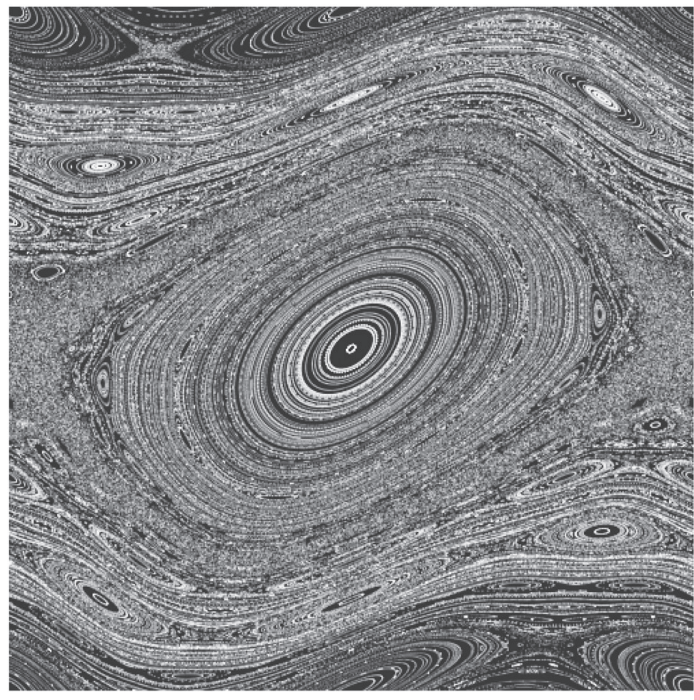

Figure 10. Some orbits of the standard map on the torus $\mathbb{T}^{2}$

on [4]. Despite his illness, he attemped one of his most ambitious courses. Weakened, however, he only could present the first two lectures - clear and deep.

\section{Conservative dynamics}

Jean-Christophe wanted to "build a theory of (weakly) hyperbolic systems that would allow, in the long run, to deal with other still poorly understood examples, in particular in the conservative." [15] He had in mind systems that preserve a volume form. The model family of maps is the standard family on $\mathbb{T}^{2}=\mathbb{R}^{2} / \mathbb{Z}^{2}$ :

$$
(x, y) \mapsto(2 x+a \sin (2 \pi x)-y, x) .
$$

The goal being to answer this well-known dynamicists' problem [12]:

Conjecture (Sinai). The standard family satisfies property (H) for a set of parameters $a$ and of points $z \in \mathbb{T}^{2}$ of nonzero Lebesgue measure.

The hypothesis of volume preservation leads to new phenomena. Indeed, the Kolmogorov-Arnold-Moser theorem shows the existence of families of closed invariant curves, whose union has non-zero volume, and such that on these curves property $(\mathcal{H})$ cannot be satisfied. Therefore it should be checked that this "elliptic" part of the dynamics can coexist with a non-uniformly hyperbolic part, or that it can even disappear when the parameter $a$ is large enough.

\section{Billiards}

In a short interview [14], Jean-Christophe explains that Sinai's conjecture is his favourite open problem in dynamical systems. He decides to talk about it in a more "concrete" example.

If one plays billiards inside a convex domain $U$, one identifies each trajectory by taking note, at each reflection, of the point of impact on the edge of $U$ and of the angle of incidence. One thus obtains a map of the ring $A:=\partial U \times(-\pi / 2, \pi / 2)$ which associates to each reflection the next reflection. It is a diffeomorphism that preserves a volume form! In the very 


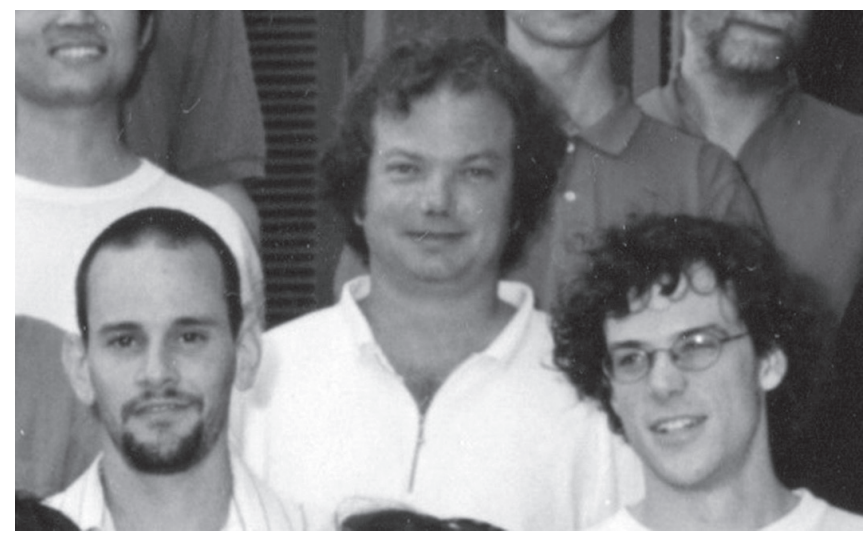

Figure 11. Jean-Christophe Yoccoz and two of his students at Cetraro, in 1998

special case where the billiard table $U$ is an ellipse, each orbit passing near the edge of the ring $A$ belongs to an invariant curve. In the general case, one expects to observe the coexistence of invariant curves and non-uniform hyperbolicity:

Problem. Show that for the map associated with a convex billiard which is not an ellipse, the set of points verifying property $(\mathcal{H})$ has non-zero volume.

Horseshoes with dimension arbitrarily close to 2 appear within conservative dynamics. Yet, when $d^{s}+d^{u}$ increases, the recurrence of the critical set tends to be stronger. To show the non-uniform hyperbolicity of these systems, it seems necessary to consider frequent compositions of fold type maps leading to higher order maps.

The difficulty is considerable. Jean-Christophe said he was not hoping for a solution before the end of the 21 st century!

\section{Credits}

The picture of figure 10 has been obtained with an interactive numerical experiment available at experiences.math.cnrs.fr and done by J.-R. Chazottes and M. Monticelli. The authors thank Stefano Marmi for the picture 11.

\section{Bibliography}

[1] M. Benedicks, L. Carleson, The dynamics of the Hénon map. Ann. of Math., 133 (1991) 73-169.

[2] M. Benedicks, M. Viana, Solution of the basin problem for Hénon-like attractors. Invent. Math, 143 (2001) 375-434.

[3] M. Benedicks, L.-S. Young, Sinai-Bowen-Ruelle measures for certain Hénon maps. Invent. Math, 112 (1993) 541-576.

[4] P. Berger, Abundance of non-uniformly hyperbolic Hénon like endomorphisms. Astérisque, 410 (2019).

[5] X. Buff, La connexité locale de l'ensemble de Mandelbrot. In: S. Crovisier, P. Berger, P. Le Calvez, C. Matheus (Eds.), JeanChristophe Yoccoz. Gazette des Mathématiciens, numéro spécial, (Avril 2018).

[6] M. Jakobson, Absolutely continuous invariant measures for one-parameter families of one-dimensional maps. Comm. Math. Phys., 81 (1981), 39-88.

[7] C.G. Moreira, J.-C. Yoccoz, Tangences homoclines stables pour des ensembles hyperboliques de grande dimension fractale. Ann. Sci. École Norm. Sup., 43 (2010) 1-68.
[8] S. Newhouse, The abundance of wild hyperbolic sets and non smooth stable sets for diffeomorphisms. Publ. Math. Inst. Hautes Études Sci., 50 (1979) 101-151.

[9] J. Palis, F. Takens, Hyperbolicity and sensitive chaotic dynamics at homoclinic bifurcations. Cambridge Studies in Advanced Mathematics vol. 35, Cambridge University Press, Cambridge, 1993.

[10] J. Palis, J.-C- Yoccoz, Non-uniformly hyperbolic horseshoes arising from bifurcations of Poincaré heteroclinic cycles. Publ. Math. Inst. Hautes Études Sci., 110 (2009) 1-217.

[11] M. Rees, Positive measure sets of ergodic rational maps. Ann. Sci. École Norm. Sup., 19 (1986), 383-407.

[12] Ya. Sinai, Topics in Ergodic Theory. Princeton Mathematical Series vol. 44, Princeton University Press, Princeton, 1994. (Lecture 13).

[13] J.-C. Yoccoz, A proof of Jakobson's theorem. Astérisque 410 (2019), 15-52.

[14] J.-C. Yoccoz, Billiard et chaos mathématique (2014). Les entretiens du Collège de France.

www.college-de-france.fr/site/jean-christophe-yoccoz/ Billard-et-chaos-mathematique-3-.htm.

[15] J.-C. Yoccoz, Résumé de cours au Collège de France (1997).

[16] J.-C. Yoccoz, Introduction to hyperbolic dynamics. In: Hillerod (Ed.): Real and complex dynamical systems. NATO Adv. Sci. Inst. Ser. C Math. Phys. Sci. 464, Kluwer, 1995. pp. 265-291.

[17] J.-C. Yoccoz, Recent developments in dynamics. In: Proceedings of the International Congress of Mathematicians (Zürich, 1994), Birkhauser, 1995. pp. 246-265.

[18] J.-C. Yoccoz, Polynômes quadratiques et attracteur de Hénon. Astérisque, 201-203 (1992). Séminaire Bourbaki, exposé 734, pp. 143-165.

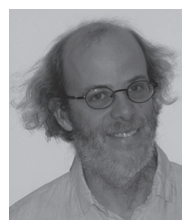

Sylvain Crovisier[crovisier@math.u-psud. fr] is directeur de recherches at the CNRS and works at the Laboratoire de mathématiques d'Orsay (Université Paris-Sud). His research deals with topological and differential dynamical systems and their perturbations.

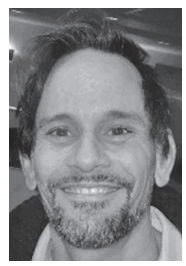

Samuel Senti [senti@im.ufrj.br] is professor at the Federal University of Rio de Janeiro, Brazil, country to which he migrated in 2004. His works deal with dynamical systems and ergodic theory. Outside mathematics he is a practitioner of "capoeira".

This is an English translation by Rafael Sasportes of the French original article entitled "Un problème pour le XXI(I)ème siècle", published in April 2018 in the special issue of La Gazette des Mathématiciens dedicated to JeanChristophe Yoccoz. The EMS Newsletter thanks the author, La Gazette des Mathématiciens, and the Société Mathématique de France for authorisation to republish this article. 